\title{
A review of epidural simulators: where are we today?
}

\author{
Neil Vaughan ${ }^{1}$, Venketesh N. Dubey ${ }^{1}$, Michael Y. K. Wee ${ }^{2}$, Richard Isaacs ${ }^{2}$ \\ ${ }^{1}$ Bournemouth University, School of Design, Engineering \& Computing \\ ${ }^{2}$ Poole Hospital NHS Foundation Trust, Department of Anaesthesia
}

\begin{abstract}
Thirty-one central neural blockade simulators have been implemented into clinical practice over the last thirty years either commercially or for research. This review aims to provide a detailed evaluation of why we need epidural and spinal simulators in the first instance and then draws comparisons between computer-based and manikin-based simulators. This review covers thirtyone simulators in total; sixteen of which are solely epidural simulators, nine are for epidural plus spinal or lumbar puncture simulation, and six, which are solely lumbar puncture simulators. All hardware and software components of simulators are discussed, including actuators, sensors, graphics, haptics, and virtual reality based simulators. The purpose of this comparative review is to identify the direction for future epidural simulation by outlining necessary improvements to create the ideal epidural simulator. The weaknesses of existing simulators are discussed and their strengths identified so that these can be carried forward. This review aims to provide a foundation for the future creation of advanced simulators to enhance the training of epiduralists, enabling them to comprehensively practice epidural insertion in vitro before training on patients and ultimately reducing the potential risk of harm.
\end{abstract}

Keywords: Epidural, Spinal, Simulator, Lumbar puncture, Haptics, Needle insertion, Modelling 


\section{Introduction}

Epidural analgesia and anaesthesia is commonly used as a form of pain relief during childbirth, for the treatment of chronic back pain or as a means to provide anaesthesia or analgesia during specific operations. An epidural is " $a$ complex amalgam of clinical judgement, technical skills, materials and equipment, drug delivery systems, patient supervision and care pathways. In addition to inherent complications in the procedure, each of these facets has the potential to generate patient harm through a combination of patient characteristics, human error or shortfalls in performance, equipment dysfunction of broader system failures. As a consequence, an enormous number of injuries can result" [1]. Data from the Royal College of Anaesthetists National Audit Project 3 revealed that from approximately 700,000 central neuraxial blocks performed annually in the NHS, $40 \%$ are epidurals. Incidence of permanent harm from all epidurals ranged from 3.1 in 100,000 to 6.1 in 100,000 [2]. Harm in these circumstances denotes vertebral abscess, haematoma and direct nerve injury. Two large studies of obstetric epidural complications report that, although rare, life-threatening complications do occur $[3,4]$. Post-dural puncture headache (PDPH), which can be extremely disabling for the mother, has a much more frequent occurrence of approximately 1\% [5]. Yet Gupta et al. identifies a potentially worrying trend in the UK of an increasing dural tap rate and quotes an incidence of $2.8 \%$. Ascribing this to the decrease in experience of trainees, the paper concludes by stating that, "we need to focus on new methods of training in epidural insertion" [6].

Changes to the training structure and compliance with the European Working Time Directive have created fewer opportunities for anaesthetists to learn technical skills in work time. Cumulative sum (cusum) analysis is a statistical and graphical tool that examines trends for sequential events over time. It has been used to determine proficiency in technical procedures. Naik et al. found that using this method, a novice attains epidural insertion competency between 1 and 85 attempts [7]. More importantly, it demonstrates that some residents may actually require as many as 75 attempts to become proficient. Konrad et al. also demonstrated that epidural anaesthesia was the most difficult practical anaesthetic skill in which to become competent, reaching a success rate of barely $80 \%$ after 90 attempts [8]. Such numbers could potentially be difficult to achieve within a defined training period.

An Australian survey of junior anaesthetic registrars learning epidural analgesia revealed that the most common teaching method was described as "guided practical experience on patients provided by a senior registrar or consultant" [9]. 
Recent reviews have commented on the poorly structured system for teaching skill acquisition and highlight the shift away from practicing on patients, even with informed consent, due to reduced tolerance for medical error [10-12]. There is increasing accountability of the medical profession and an increased emphasis on patient safety [13]. A recent review of claims handled by the National Health Service Litigation Authority (NHSLA) between 1995-2007 revealed that 366 of 841 cases $(44 \%)$ were related to regional anaesthesia and of these, half arose in the obstetric setting [14]. Examples of claims included neurological damage following labour epidurals, dural puncture leading to headache and backache, and pain during labour and caesarean section. This not only highlights the potential morbidity from an epidural but also the substantial cost burden to the NHS.

Epidural simulators have been marketed with claims to provide the trainee with a similar feel to that encountered in vivo. The procedure of inserting an epidural Tuohy needle into the lumbar spine requires the operator to visualise in their mind a three-dimensional (3D) anatomical image of the bony structures and the various tissue layers from skin, through to subcutaneous fat, supraspinous and interspinous ligaments, ligamentum flavum and then to the epidural space. Epidural needle insertion is essentially a blind procedure, but utilises a well-known technique referred to as "loss of resistance" (LOR). First described by Dogliotti in 1933, LOR essentially involves identification of the epidural space by compression of either fluid or air as the epidural needle encounters the various ligaments and potential spaces of the lumbar vertebral column [15]. Initially, the back of the patient is palpated, and using surface landmarks such as the iliac crests, an assessment is made of suitable intervertebral spaces and of midline. For lumbar epidurals, this may be between lumbar vertebra 3 (L3) and lumbar vertebra 4 (L4) for instance. The epidural or Tuohy needle, as it is commonly called, is inserted into the interspinous ligament and a syringe filled with saline is attached to the end of the needle. These LOR syringes are specially manufactured so that there is little friction between the plunger and the inside of the syringe. A constant or intermittent force is then applied to the plunger by the operator's thumb as the needle is slowly advanced forwards. As the tougher and more fibrous ligamentum flavum is encountered, a higher resistive force to injection is encountered. Once the needle tip traverses the ligamentum flavum, the epidural space is then entered into and saline can be quite easily injected, hence the phenomenon of LOR. It is this haptic perception and feedback that informs the operator of needle location within the various tissue layers, obstruction from bone and loss of resistance from potential spaces. Combining this with the creation in one's mind of a three-dimensional image of lumbar spinal anatomy enables successful placement of an epidural catheter. 
An alternative technique, often used when siting thoracic epidurals, is the paramedian approach. Due to the steep angulation of spinous process in the thoracic region and less dense ligaments, difficulty with insertion or a false LOR is not uncommon and so many epiduralists prefer this other approach over midline entry for thoracic epidurals. The needle is initially inserted $1-2 \mathrm{~cm}$ lateral to the spinous process and then advanced perpendicularly until the lamina of the vertebra is encountered. The needle is then reangled and advanced further so that the needle 'walks off' the lamina and approaches the ligamentum flavum. The LOR technique is then employed.

The lumbar puncture procedure is slightly different to an epidural insertion in that a spinal needle is inserted between the lower lumbar vertebrae and advanced further so that it penetrates the ligamentum flavum. A 'pop' is felt which indicates that the needle has punctured the dura mater which is a thin membrane enclosing the spinal cord and subarachnoid space. The stylet from the spinal needle is then withdrawn and cerebrospinal fluid (CSF), which leaks out from the needle, can be sampled or local anaesthetic and pain relieving drugs can be injected for anaesthesia and analgesia.

Spinal anaesthesia differs from epidural anaesthesia not only in technique but also in clinical effect. During an epidural, a catheter tube is inserted into the epidural space allowing additional doses of local anaesthetic to be administered if required or an infusion to be commenced, whereas a spinal is a single-shot technique with no extraneous material left insitu. The onset of analgesia is approximately 15-30 minutes following an epidural, whereas it is approximately 3-5 minutes following a spinal anaesthetic. The injected dose is usually about ten times more when given down an epidural. Epidurals may be inserted at lumbar, thoracic, or cervical levels, whereas a spinal must be injected below L2 to avoid trauma to the spinal cord.

During an epidural insertion, the operator tries to perceive which tissue layer the needle tip is passing through by feeling the resistances on the needle. This is a process known as "haptic feedback". A simulator can thus assist the development of this visiospatial awareness of spinal anatomy and 'feel' of the procedure to allow practice prior to attempts on patients. Not only will this serve to enhance patient safety but it also creates a safe and controlled environment in which to learn. The value of simulation in performance analysis of any practical skill has been demonstrated by Wong et al. in a manikin study of needle cricothyroidotomy [16]. The publication "Consent for Anaesthesia" produced by the Association of 
Anaesthetists of Great Britain and Ireland, emphasises the need to minimise harm to patients and suggests initial manikin practice [17]. The new curriculum for a Certificate of Completion of Training (CCT) in Anaesthetics by Royal College of Anaesthetists states that "the historical reliance upon workplace-based learning is being compromised further by decreasing hours spent in the clinical setting. This and other factors combine to encourage the use of simulation to enhance learning in anaesthesia" [18]. Simulation is gaining popularity not only for the purpose of creating mock scenarios but also in learning psychomotor skills [19-23].

The accuracy of the forces in epidural simulators is a topic which is under recent discussion [24-26], so it is important that the forces required to insert a needle during simulation match those achieved in reality. Skills learned during this simulation can then be transferred to the actual clinical environment.

Since the early 1980's epidural simulators have been developed both in academic institutions and commercially, the common goal is to aid the practical skills of clinicians; predominantly anaesthetists. Since this time, there have been substantial advances in the field of simulation in anaesthesia. Reviews of certain epidural simulators have been conducted but not comparative studies covering the majority of existing simulators. One such review by Gillespie et al. compared a physical and a perceptual method for creating epidural simulators and found the perceptual model to be superior [27]. Friedman et al. compared high-fidelity versus low-fidelity epidural simulators [28]. The study compared how much each simulator advanced skill level in novice anaesthetists and found no differences between the high or low-fidelity epidural models and suggested that low-fidelity versions may be more cost effective. Other reviews [19-23] have gathered opinions about epidural simulators amongst anaesthetists and generally reported very positive feedback.

This review aims to compare epidural simulators but also highlights lumbar puncture simulators for both adult and paediatric practice. It covers thirty-one simulators in total; sixteen of which are solely for epidurals, nine also include spinal or lumbar puncture, and six solely lumbar puncture simulators. The focus will be on the technologies used, implementation methods and capabilities that each simulator provides. This includes the designs of hardware, actuators, sensors, haptic devices and the software, virtual reality, stereographics, 3D vertebrae and virtual patients that have been used in epidural simulators. The simulators are categorised into computer-based and manikin-based simulators. The computer-based simulators are technologically advanced, whereas manikin devices are based on physical models without 
any electrical or mechanical control. A patent search was conducted on a worldwide patent database via the European patent office (EPO) and patents related to epidural simulation were researched. All existing simulators are compared to identify the strengths and weaknesses of each model. By drawing these together, we will suggest the ideal characteristics for an epidural simulator to assist in future development.

\section{Tuohy needle insertion forces}

Epidural needle insertion consists of a complicated interaction of many forces: a) each tissue present has various viscosity, elasticity, density and frictional properties b) bubbles of air in saline can compress increasing compliance c) the method of insertion can vary depending upon needle inclination angle, paramedian angle, speed of insertion and twisting of the needle (torque) d) properties of the needle can vary, including the angle of the tip, tip type - side tipped or two-plane symmetric, needle gauge from $15-20 \mathrm{G}$ and width of the metallic walls in hollow needles vary e) plunger resistance is caused by friction on the syringe walls f) the flow of saline is restricted by the outlet of the syringe and finally, g) the needle orifice can be obstructed with tissue reducing cutting and saline release.

It is not feasible to measure all of these forces individually in vivo and it would not make sense to measure the exact proportions of each force that make up the reaction force. In practice, it may be sufficient to measure the resultant pressure of the saline instead. Measuring resultant pressure provides an indication of all reaction forces over the plunger area, which is felt by the anaesthetist during insertion. This combination of all forces must be able to be replicated in order to simulate the feeling of needle insertion.

Theoretically, a model can represent the resultant force down into its constituents. The thumb applies force onto the plunger of the syringe and this force causes the saline to get 'resultant pressure' (Eq. 1), see Fig. 1. This pressure provides the 'feel' as the needle is pushed forwards. Anaesthetists tend to hold the 'wings' of the epidural needle to stabilise the needle and also apply a forward force during needle advancement, however, the actual feel for LOR comes from the plunger push which is seldom used in conjunction with wing push. This generates the 'reaction force' which is equal and opposite to the applied force and comprised of several factors; a) the cutting force required for the needle tip to pierce the tissue b) friction caused by needle shaft rubbing on the tissue c) static friction to get the stationary needle moving d) side 
compression force is caused by the surrounding tissues e) torque is caused by twisting of the needle and f) all of these forces vary according to depth and tissue stiffness.

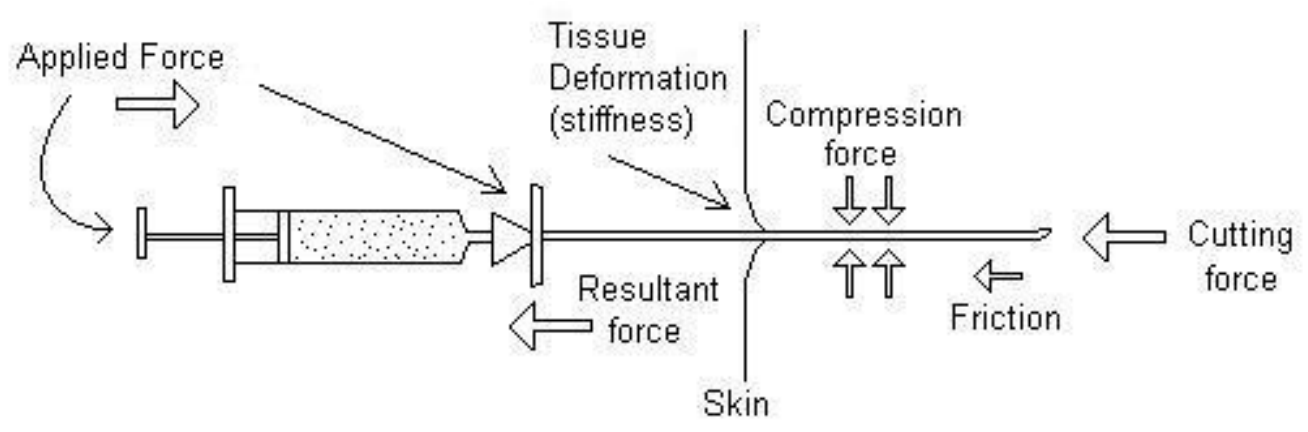

Figure 1. Several forces involved with needle insertion

In principle if the applied force becomes instantaneously greater than the reaction force this means the needle is moving forwards, otherwise the needle is assumed to be stationary in relation to the tissue surface causing tissue deformation (Eq. 2). The resultant pressure still varies with applied force whether the needle is moving or stationary.

Resultant pressure of saline, $P=(F z / A)$

where $F z$ is thumb force on plunger, $A$ is area of plunger.

The needle moves forward only when the applied force is greater than the reaction force momentarily.

$$
\begin{aligned}
& \text { Velocity }=\{0, \quad F z=F r \\
& \text { v, } \mathrm{Fz}>\mathrm{Fr}
\end{aligned}
$$

where $F z$ is applied force (force on plunger), $F r$ is the reaction force and $v$ is needle velocity.

Reaction force has many components, but the main three are cutting force, friction and stiffness of tissue and the needle combined (Eq. 3) [26]. So the reaction force comprised of, Cutting force + frictional force + stiffness force

$$
F r=F c+F_{f}+F s
$$


Static friction only occurs when the needle is not moving and it increases with applied force and tissue depth (Eq. 4).

$$
\begin{aligned}
\text { Static friction }=\{f(F z), & v=0 \\
0, \quad v & >0
\end{aligned}
$$

where $f(F z)$ is a force model based on applied force $F z$ and tissue depth, $v$ is needle velocity.

The stiffness force occurs when applied force is greater than zero but has not pierce through the tissue (Eq. 5). When the needle is stationary, stiffness force increases with applied force until reaching a maximum value for each layer, when the needle pierces the tissue and starts to move forwards this results in cutting force (Eq. 6).

Stiffness force $F s=k^{*} x$,

where $k$ is the tissue stiffness and $x$ is the tissue deformation

$$
\begin{aligned}
& \text { Cutting force } F c=\left\{\min \left(F z-F_{f}, 12.9\right), \quad d \leq 3\right. \\
& \min \left(F z-F_{f}, 6\right), \quad d \leq 9 \\
& \min \left(F z-F_{f}, 9\right), \quad d \leq 13 \\
& \min \left(F z-F_{f}, 8\right), \quad d \leq 39 \\
& \min \left(F z-F_{f}, 11\right), \quad d \leq 42 \\
& \min \left(F z-F_{f}, 0\right), \quad d \leq 48 \\
& \min \left(F z-F_{f}, 2\right), \quad d>48
\end{aligned}
$$

where $d$ is needle tip depth in $\mathrm{mm}, F z$ is applied force, $F_{f}$ is frictional force, function min() returns smallest of two numbers. Values are in Newtons, based on the data collected form the porcine trial (the insertion force in porcine model is considered to be higher than that in humans) as shown in Table 1.

Friction also occurs when the needle is moving (Eq. 7), which is when the cutting force is at its maximum.

$$
\text { Friction } \begin{array}{rlr}
F_{f}=\{F r-F c, & v>0 \\
0, & v=0
\end{array}
$$

Where $F r$ is reaction force, $F c$ is cutting force, $v$ is needle velocity 
Simulators should accurately represent the thickness, depth, and reaction force $(F r)$ of each tissue layer. There are studies reporting the thickness and depth of ligamentum flavum, epidural space and supraspinous ligament [29-32]. Also there are studies reporting the force required for a needle to penetrate the various ligaments in porcine cadavers [33, 34]. However, during pregnancy tissue properties may change due to excess water accumulating in tissues.

\begin{tabular}{|l|l|l|l|}
\hline Porcine Tissue Layer & Thickness $(\mathrm{mm})$ & Depth $(\mathrm{mm})$ & Insertion force $^{1}(\mathrm{~N})$ \\
\hline Skin & 3 & 0 & 12.9 \\
Subcutaneous fat & 6 & 3 & 6 \\
Supraspinous ligament & 4 & 9 & 9 \\
Interspinous ligament & 26 & 13 & 8 \\
Ligamentum flavum & 3 & 39 & 11.1 \\
Epidural space & 6 & 42 & 0 \\
Dura & 15 & 48 & 2.0 \\
\hline
\end{tabular}

Table 1. Various properties of each tissue layer in porcine cadavers [34]

\section{Epidural simulators}

\subsection{Manikin-based epidural simulators}

Manikin epidural simulators are based upon physical manikin or dummy models made from plastic or rubber. They are not computer-driven and generally contain no mechanical or electronic parts or technology. They are portable, easy to set up and use and have physically palpable anatomical landmarks. The 'Greengrocer Model,' originally proposed by Leighton in 1980, is probably one of the simplest and cheapest epidural simulators that has ever been developed [35]. It consists of a foam block containing bread and a banana which represents the skin, subcutaneous tissue and ligamentum flavum (Fig. 2a). The average banana can be used about thirty times. A balloon hidden inside represents the dural layers and its rupture is used to indicate a dural tap. This simplistic model can assist as part of a perceptual training course. However this clearly does not recreate an accurate feeling of human tissue. There is no 'bone' so the needle will penetrate through at any angle or position.

More recently, several simulators based on plastic human-shaped models have been developed (Fig. 2b-c). These often contain solid structures to represent the lumbar vertebrae. The surface is covered with an artificial synthetic skin, which is

\footnotetext{
${ }^{1}$ This is saline pressure divided by the plunger cross-sectional area
} 
often replaceable with various densities to represent patient variation. There is often a composite rubber spinal canal, filled with a liquid to mimic CSF. If punctured with a needle, some may emit an audible 'pop', followed by a CSF leak as occurs in vivo. Examples commercially available include the Thoracic \& Lumbar Epidural Anaesthesia Simulator (Limbs and Things Ltd, Bristol, UK), manufactured by Kyoto Kagaku Co. Ltd, Japan [36], Lumbar Epidural Injection and Lumbar Puncture Trainer, (Pharmabotics Ltd, Hampshire, UK) [37], Adult Lumbar Puncture Simulator (Shengyuan Medical Devices Co., Ltd) and Adult Lumbar Puncture Simulator (Medi-Tech, China).
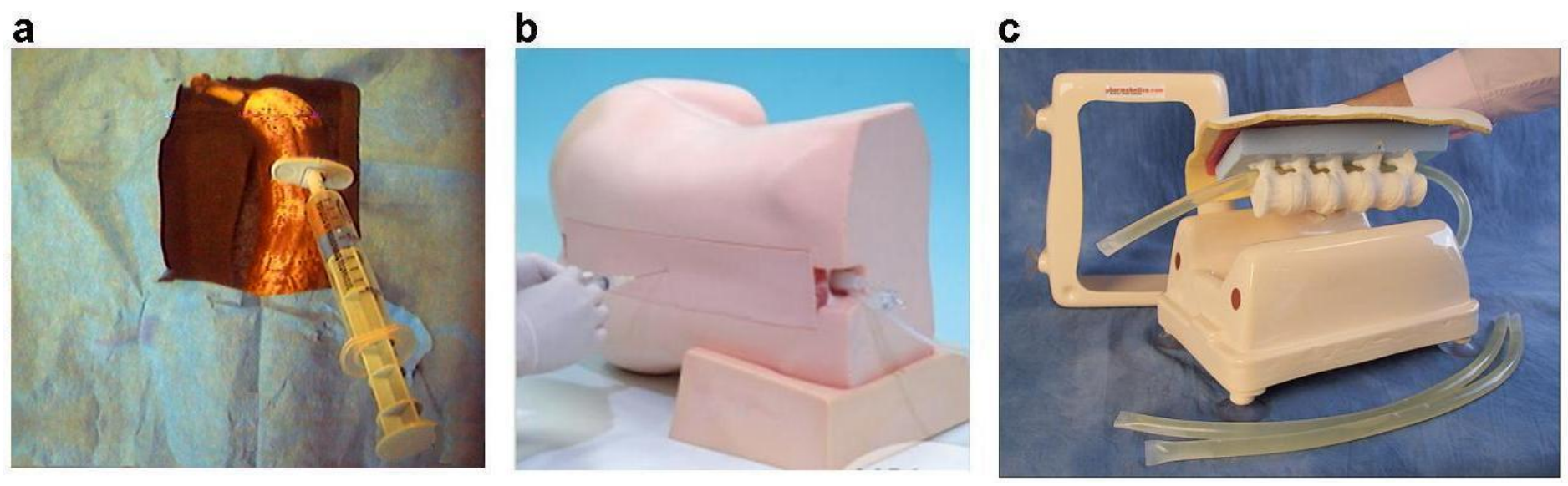

Fig. 2. Several different manikin-based simulators: a) Greengrocer model b) Thoracic \& Lumbar Epidural Anaesthesia Simulator reproduced with permission from Limbs \& Things, Bristol, UK ( 2012 c) Lumbar Epidural Injection and Lumbar Puncture Trainer, reproduced with permission from Pharmabotics Ltd.

The Thoracic \& Lumbar Epidural Anaesthesia Simulator is designed solely for epidural practice. A large section of the vertebral column is palpable from T7 to L5, including the iliac crests and inferior angle of the scapulae. The model emits a 'pop' during passage of the needle and supports both lateral positions. The Lumbar Epidural Injection and Lumbar Puncture Trainer can again be used in the lateral or sitting position. There are five palpable lumbar vertebral bones L1-L5 and the model can be disassembled to reveal the needle location as placed by the epiduralist. If accidental dural puncture occurs, simulated CSF will appear in the syringe. It supports epidural anaesthesia, lumbar puncture and spinal anaesthesia and the manufacturers state that greater than two hundred insertions can be undertaken before needing replacement. The Shengyuan Medical Devices Co., Ltd Adult Lumbar Puncture Simulator is designed for training in epidural insertion, lumbar puncture and spinal anaesthesia. It only supports the left lateral position. CSF will flow out if a dural puncture occurs. Internal vessels and pads need replacing when worn. It does not support any patient variation such as obesity. Finally, Medi-Tech's Adult Lumbar Puncture Simulator allows practice of epidural insertion plus other lumbar and spinal insertions. Again, CSF will flow out if dural puncture occurs and pads need replacing when worn. 
The Lifelform® Spinal Injection Simulator (Nasco, Fort Atkinson, Wisconsin, USA) supports epidural analgesia, spinal anaesthesia, lumbar puncture, caudal analgesia, sacral nerve block, and lumbar sympathetic block (Fig. 3a), [38]. It has a changeable fluid-filled vertebral column and replaceable skin. The insertion can be performed in either the sitting or lateral position. Lumbar vertebrae L1 and L2 are visible to assist with the teaching of spinal anatomy, with L3-L5 and the sacrum being the functional section of the simulator with clearly palpable landmarks. One newer simulator is the $M 43 B$ Lumbar Puncture Simulator-II (Fig. 3b), manufactured by Kyoto Kagaku Co. Ltd Japan in partnership with Keio University Medical School, Tokyo, and currently marketed in the UK by Limb \& Things Ltd, Bristol [39]. It allows for epidural and spinal insertion. Consisting of a lower torso manikin, it comes with removable 'skin' that represents adult, obese or elderly anatomy and a replaceable puncture block. Lateral and sitting positions as well as palpation are supported. The needle is positioned manually and the spine contains fluid to represent CSF. CSF collection as well as the measurement of CSF pressure can be practiced. However, only lumbar vertebrae L2-L5 are present, which is less than some other simulators. A recent study by Uppal in 2011 aimed to assess whether experienced anaesthetists thought that this simulator might prove to be a useful tool for training novices to perform neuraxial procedures [40]. The overall impression was very positive yet the lowest ranking aspect of the M43B simulator was the disappointing 'feel' of the ligamentum flavum. One of the most difficult aspects of epidural insertion to teach is the idea of loss of resistance (LOR) on reaching the epidural space as there may be false positives during insertion. Although most anaesthetists in this study believed the LOR to be life-like, they felt that the ligamentum flavum was "less dense and gritty" than expected and was less thick. Threading the epidural catheter was also deemed to be more challenging than usual.
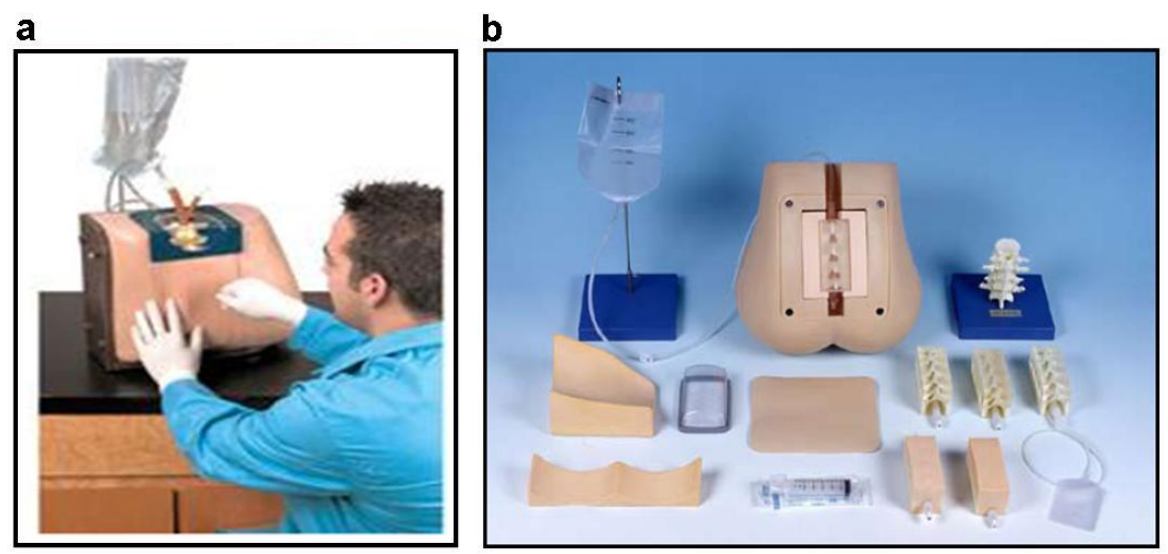

Fig. 3. Two commercially available manikin epidural simulators: a) Life/form® Spinal Injection Simulator re-produced with permission from Nasco (C) 2012. b) M43B Lumbar puncture simulator-II, reproduced with permission from John Wiley \& Sons (C) 2011 [39] and Kyoto Kagaku Co. Ltd @ 2012 . 
A few manikin simulators have incorporated some unique features, such as those that are amenable to ultrasound (US) scanning. This allows the internal structures to be viewed pre-puncture and an estimate made as to the depth and location of the epidural space. This is a potential advantage because the National Institute for Health and Clinical Excellence (NICE) recommends that although "evidence on ultrasound-guided catheterisation of the epidural space is limited in amount, it suggests that it is safe and may be helpful in achieving correct placement” [41]. The Lumbar Puncture / Epidural Manikin Trainer (Simulab Corporation, Washington, USA) is ultrasound compatible and contains palpable lumbar vertebrae with spinous processes and iliac crests (Fig. 4a), [42]. Ligamentum flavum, dural sac and the epidural space are present. Interchangeable tissues provide support for obese, normal, elderly and elderly obese. Simulation CSF will leak out to indicate a dural puncture. The Blue Phantom Lumbar Puncture and Spinal Epidural [43] is a manikin designed with emphasis on support for ultrasound scanning (Fig. 4b). It can be used with any standard US probe with normal settings and the internal landmarks will be visible. It supports sitting upright or lateral decubitus. The spine can be palpated and the needle location can be anywhere, to target cervical, lumbar or thoracic region. Obese spinal inserts are available with thicker tissue. Lumbar puncture, epidural and other procedures are supported. CSF will leak out from the spinal canal. The spine tissue modules contain spinal segment, skin tissue, ligamentum flavum, epidural space, dura, subarachnoid membrane, and subarachnoid space containing cerebral spinal fluid. The Adam Rouilly Regional Anaesthesia Simulation Manikin is a complete upper body manikin which supports epidural insertion at all vertebral levels including many other nerve blocks (Fig. 4c), [44]. It contains a specially coated X-ray compatible spine. Only the head is covered with artificial skin, the body is covered with cloth. Developed by the Manchester and Salford Pain Centre primarily for nerve blocks for pain management, the unique feature with this device is the added support for radiological X-ray screening to confirm needle placement. The density of the manikin is low so that the doses of radiation are reduced during the simulated procedures. 
a

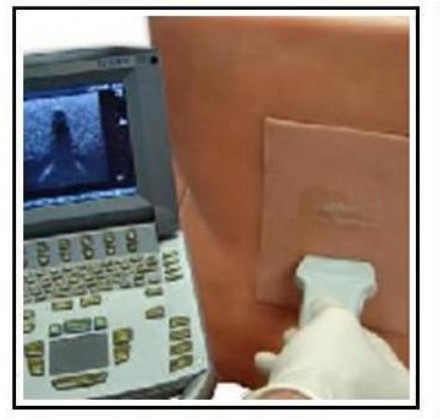

b

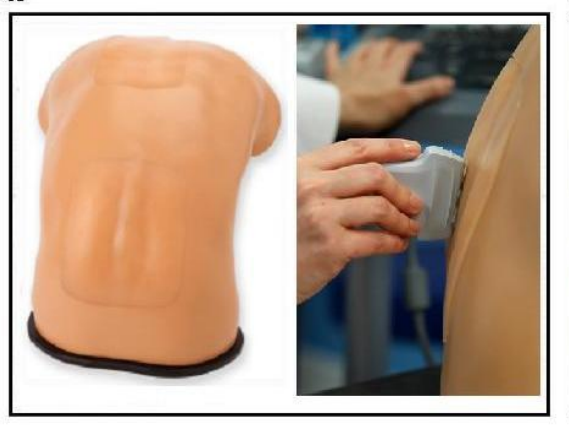

C

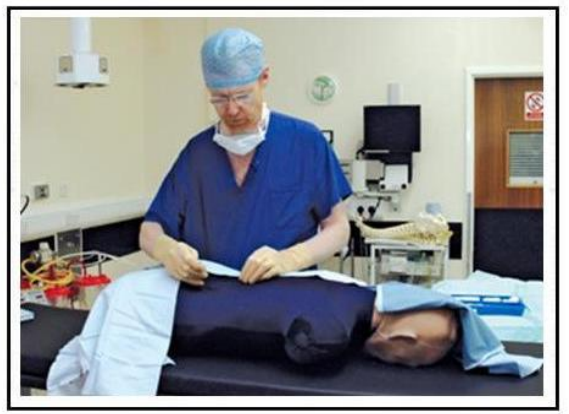

Fig. 4. Manikins with ultrasound or X-Ray support. a) Simulab Lumbar Puncture Trainer reproduced with permission from Simulab @ 2012. b) Blue Phantom Lumbar Puncture and Spinal Epidural Simulator, reproduced with permission from Blue Phantom. c) Adam,Rouilly Regional Anaesthesia Simulation Manikin, reproduced with permission from Adam,Rouilly @ 2010.

In 2003, the Epidural Simulator Training Apparatus (ESTA) was designed using carbon-fibre electrically-conductive fabric in between artificial tissue layers. The fabric (Gorix) allowed electrical detection of needle location without a wire being attached to the Tuohy needle. The production of ESTA have been discontinued due to the difficulty in sourcing Gorix.

Patents have been filed for epidural simulators. A simulator device patented by Duggan attaches to the desk with a clamp and uses torso-shaped layers of high density polyethylene with foam in-between (Fig. 5), [45]. The closed cell architecture of the foam resists the injection of air and saline. Another device patented by Kurosaki consists of layers of synthetic material which represent skin, tissues and ligaments which can be penetrated by the needle and bone areas that cannot be penetrated [46]. A manikin simulator patented by Zhangji and Baoquan is made from PVC (Polyvinyl Chloride) and is able to bend at the waist to increase the intervertebral space before the needle is inserted [47]. In reality, prior to epidural insertion, the patient assumes a bent over posture for this very reason, thus enhancing the chance of success. 


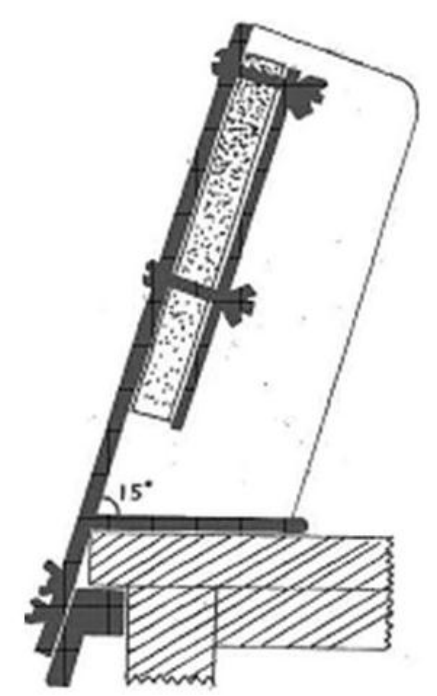

Fig. 5. An epidural simulator with a layer of closed-cell foam and a $75^{\circ}$ patient position was patented by Duggan (2000).

\subsection{Computer-based Epidural Simulators}

This section contains mechanical, electronic or computer based epidural simulators. These often include haptic devices, computer controls or virtual reality. The first mechanical simulator was created in 1990 by Daykin and Bacon and named the Epidural Injection Simulator [48]. It works by inserting a Tuohy needle through a spring-loaded device. This provides frictional resistance forces similar to those of tissues on a needle. At the certain depth, corresponding to the average distance of skin to the epidural space, the needle triggers a solenoid valve to open and saline escapes to simulate loss of resistance. This was a relatively simple device without computer control. Langton and Meiklejohn were probably one of the first groups to measure the pressures exerted during insertion of lumbar epidurals in obstetric patients [49]. The readings obtained were then compared with those from the aforementioned epidural injection simulator. It was found that there were no differences between that of the simulator and those measured in vivo. This was probably one of the first studies to actually put a simulator to the test.

a

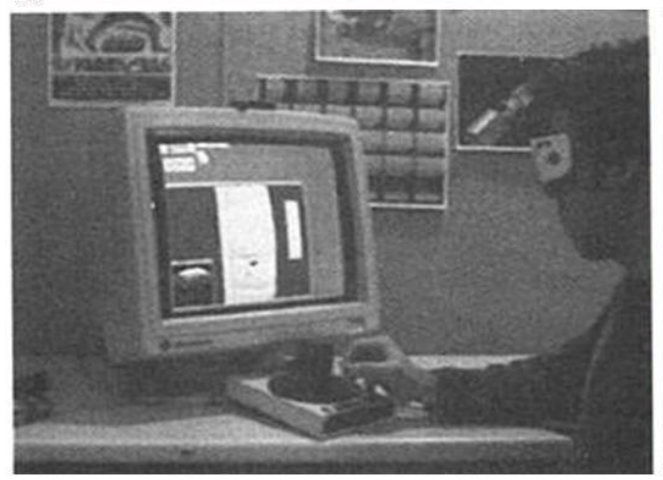

b

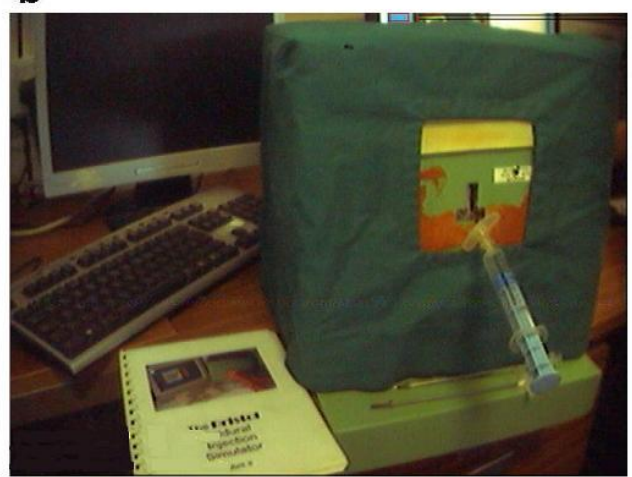

C

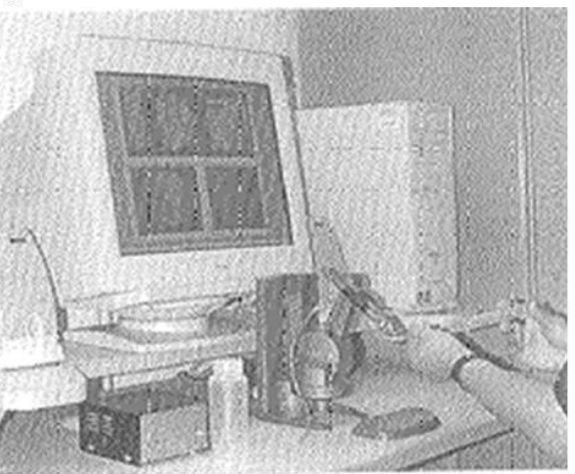

Fig. 6. Early computer-based simulators allow haptic devices connected with real needles: a) Stredney et al. (1996) [49] b) Bristol (1998) c) Dang (2001). 
In 1996, Stredney et al. made a considerable contribution with the first computer-controlled haptic epidural simulator [50]. The needle is moved by an Impulse Engine 1000 (Immersion Corporation) haptic device, attached to an actual needle. The computer displays a 3D patient model with a vertebral column and a polygon model of the needle. The 3D patient model data came from magnetic resonance images (MRI) scans of actual patients. Haptic forces are based on data obtained during epidural insertions on porcine and canine cadavers combined with expert opinion. There are multiple data sets representing various body types. CrystalEyes ${ }^{\mathrm{TM}}$ LCD shutter glasses are used to create an illusion of the scene in 3D (Fig. 6a). Users can view the needle position overlaid onto real MRI images. It was evaluated by anaesthetists who perceived the device to feel similar but inaccurate and 'too mechanical' compared to real insertions [50]. However, they felt positive that after refinement the device would be more realistic. The software is controlled by two-way voice recognition, allowing the user to activate the simulation by speaking commands into a microphone, and the computer generates voice feedback through speakers. Speech recognition is unique to this simulator and an advantage since the computer interface is simplified since no text is required on screen. The lack of a physical manikin means that the spine is not palpable and the needle insertion position is only chosen via the visual display unit.

Brett et al. created an epidural simulator whereby the haptic forces were based on measured forces of epidural needle insertion from the skin to the epidural space [51]. The needle was fitted with a strain gauge to infer the applied force. During simulation, feedback force is calculated based on which tissue the needle is traversing from a mechanicallyderived tissue model. The haptic forces are generated from a single-axis actuator mechanism attached to a Tuohy needle. The data used was based on human tissue samples not from in vivo experiments

In 1998, the Bristol epidural simulator was introduced by University of the West of England (Burrough House Associates) and is still currently used by several hospitals in the UK (Fig. 6b). The mechanical device box exerts force feedback on the needle and simulates loss-of-resistance when the epidural space is located. Paramedian insertion is not supported, but upward inclination angles are supported. A hydraulic valve is controlled based on defined geometry and structures allowing saline to inject inside the box when the epidural space is reached to mimic the in-vivo experience. The computer screen shows a $2 \mathrm{D}$ cross-section of the spine and indicates the needle position. The device can be connected to a standard 
PC or laptop. The instruction manual gives details about how to replace damaged needles, use saline or air, how to attach new skin patches. Scrollbars in the software allow selection of the resistance of each tissue.

Dang et al. created an epidural simulator which uses the Sensable Phantom haptic device (SensAble Technologies, Massachusetts, USA) [52]. The LOR syringe is connected via a solenoid valve which allows saline to flow out to simulate loss of resistance (Fig. 6c). Here again, the forces encountered are based upon expert opinion. Tissue resistances can be further adjusted using scrollbars. The patient position can be sitting or prone. The simulator also includes a training feature called "Haptic Guidance" that allows the user to follow a previously recorded expert procedure and feel the encountered forces. The simulator was scored by expert anaesthetists as 6.4 out of 10 for realistic forces.

The Epidural Injection Simulator (EIS) (Flinders Meditech Pty Ltd, Adelaide, Australia) combines a physical interface with a virtual-reality display of needle advancement and aims to replicate human anatomy for various scenarios (Fig. 7a), [53-54]. Advantages of this model include a realistic appearance with options for alternate body shapes and skin tones and the presence of palpable anatomical features such as vertebrae and iliac crests to provide tactile feedback. It can simulate loss of resistance using air or saline techniques with 16 and 18 gauge needles. A graphical interface with real-time visual feedback indicates needle progression through each tissue layer and settings for patient variance in tissue density and depth characteristics can be adjusted. It is one of the more expensive simulators currently available on the market.
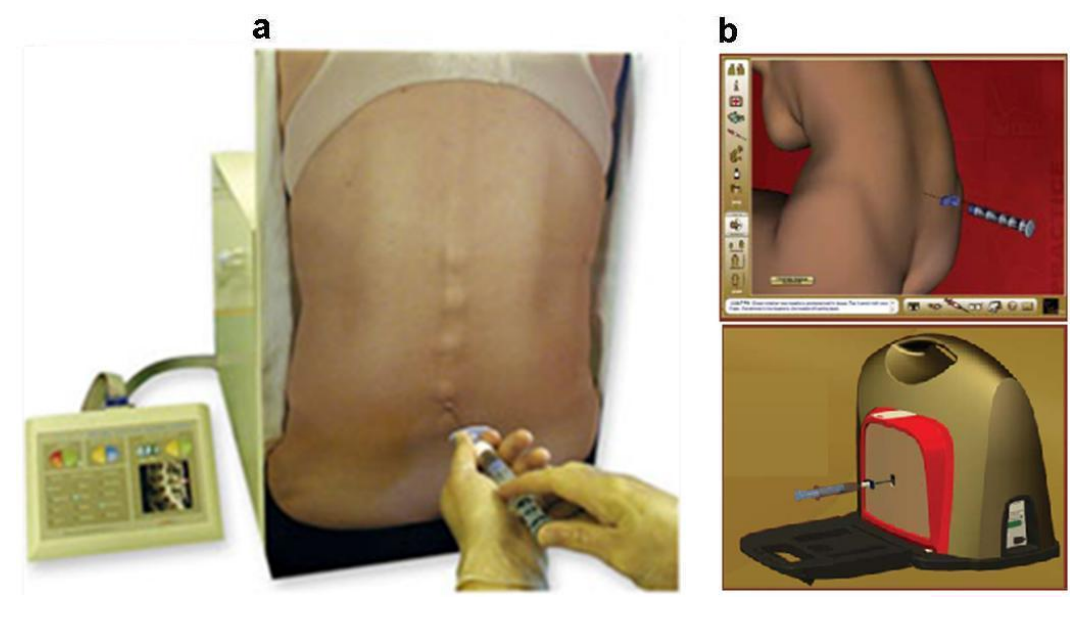

Fig. 7. Recent computer-based epidural simulators: a) Epidural Injection Simulator EIS (2003) [53-54] b) Mediseus (2006) [55-57]. 
In 2006, Mediseus ${ }^{\circledR}$ epidural simulator (MedicVision Pty Ltd, Melbourne, Australia) was launched commercially, although recently MedicVision has ceased trading [55]. Computer graphics display a complete human body with rotate and zoom functions (Fig. 7b). The skin can be transparent to reveal five modelled vertebrae. A portable box, patented by Brien and Horley, concealing a phantom omni gives force feedback on the needle and can measure air pressure inside the syringe [56]. The needle moves on screen in real time. The system gives user feedback after needle insertion. The user interface contains many options and settings, combined with the 3D graphics. There is a guide option which gives clinicians a tour of epidural anatomy. A virtual expert option displays video guidance throughout and the virtual patient asks questions which require responses. The haptic device is easily portable and can plug into a standard laptop. A review by Elks and Riley found that $37 \%$ of users rated the model's skin as realistic. Surface anatomy and back shape were considered unrealistic. $42 \%$ percent found the tactile feel realistic, 54\% rated the loss of resistance to air (LORTA) and needle grip as realistic [57].

The Spinal Anaesthesia Simulator was designed by Driefaldt et al. and Albert et al. at Limerick and Cork Universities [58, 59]. Epidural insertion and spinal anaesthesia can both be practiced. A Sensible Technologies Phantom Desktop haptic device gives insertion force feedback. A haptic immersive workbench is used with an angled screen (Fig. 8). The needle moves in $3 \mathrm{D}$ by following movement of the stylus. The virtual model of a human back is made from a combination of computerised tomography $(\mathrm{CT})$ and MRI scans which adds an extra element of realism. A control panel allows users to adjust levels of difficulty, opacity of the skin. Since this model is 'virtual', there are no tracks or marks from previous insertions and as such the number of attempts are limitless. A unique point is that Knowledge Space Theory (KST) is applied to assess the learning and skill level of trainees on many competency levels, which can aid the device's use as an assessment tool. 


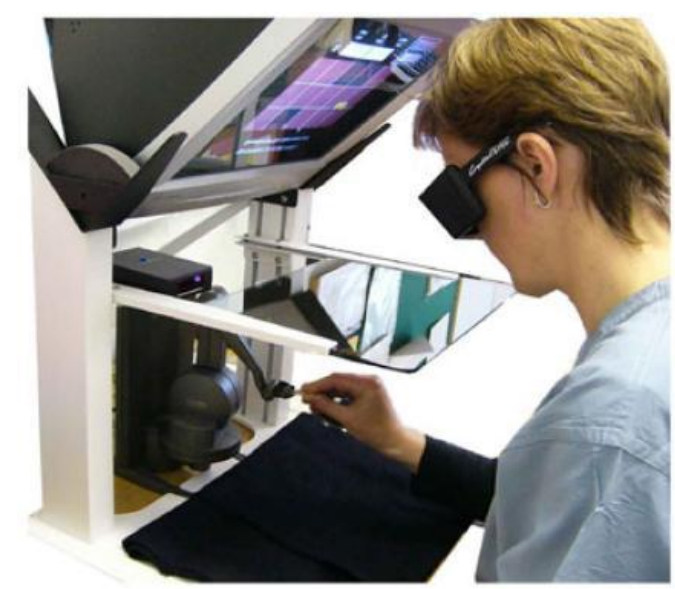

Fig. 8. Spinal Anaesthesia Simulator (2006) combines a haptic device with stereo 3D glasses. Image reproduced with permission from Springer (C) 2012 [59].

Around 2008, EpiSim was developed by The Touch Lab, Massachusetts Institute of Technology and made commercially available by Yantric Inc., Massachusetts [60]. It uses a Sensible Phantom Premium 1.0 haptic force feedback device in conjunction with a manikin and epidural needle with an actual syringe attached to recreate a loss-of-resistance sensation [61]. A computer based graphical display using virtual reality shows two vertebrae and the needle can be moved between them in real time. The bones can be viewed from two fixed angles or through a simulated fluoroscopic X-ray image. Thickness of each tissue layer can be adjusted using scrollbars, saved and re-loaded. Green and red colours on the image indicate the current tissue or warnings if bone is touched respectively. Audio vocal warnings are sounded if dura is punctured. A unique feature is the ability to record an 'expert' insertion for future playback with force feedback history incorporated. Furthermore, in the event of a problem or difficulty, it is possible to rewind the recording and assess the likely contributory factors. The combination of manikin and virtual reality gives this simulator the best of both worlds in terms of a physical model as well as quantitative information from the adaptable virtual model over any others existing simulators on the market.

\subsection{Lumbar Puncture Simulators}

Performing a lumbar puncture requires a similar level of knowledge and skill as that for epidural and spinal anaesthesia. Thus, the technology utilised here could equally be applied to future epidural simulators. Bostrum et al. created a lumbar puncture simulator which was computer-based, utilizing high-resolution 3D graphics and a force-feedback device [62]. 
The simulator consisted of a combination of specialized force-feedback needle actuator hardware, and custom highresolution graphics. Position sensors track the insertion angle or trajectory of the device, while a programmable motor or actuator provides variable resistance to insertion, depending upon which simulated anatomic structures the virtual needle is penetrating. The virtual needle can be inserted at any desired angle and resistance is generated for that angle. If an incorrect trajectory is used and bone is contacted, the resulting sudden sharp increase in resistance would be conveyed. Gorman et al. created a lumbar puncture simulator which uses a lumbar puncture needle attached to a Sensable technologies Phantom Premium 1.5 haptic feedback device (Fig. 9a), [63, 64]. A manikin is present with the haptic stylus inside. It has a graphics display showing the vertebrae and the moving needle position in 3D. Six tissue layers are modeled: skin, dermis, subcutaneous tissue, muscle, ligamentum flavum, and the dura mater. Haptic feedback varies depending upon the material in the layer model, the depth of insertion, and the insertion angle of the needle. The forces also change when the needle is initially inserted or punctures through the skin. If the needle contacts bone, the user experiences stiffer resistant forces. The spine is modeled from L1 - L5 and the sacrum. The system records each insertion and can playback what happened internally, which could assist a user's understanding during training. The spine is not palpable on the model.
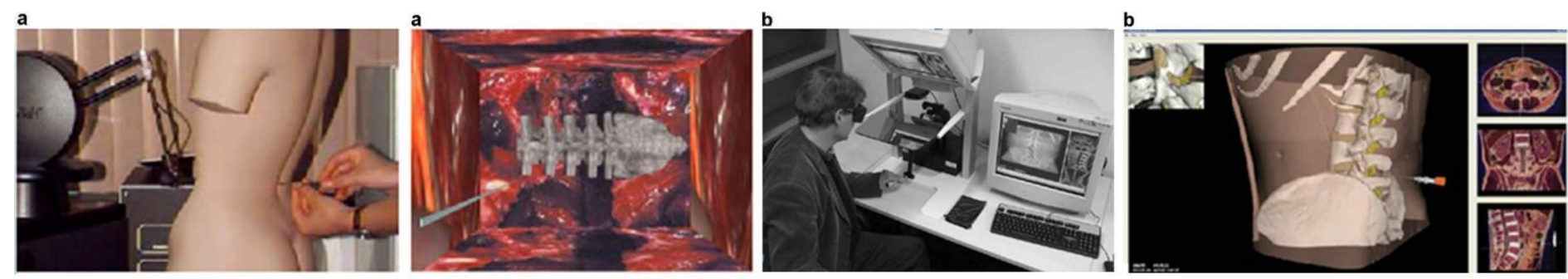

Fig. 9. Lumbar puncture simulators: a) Gorman (2000) combined a manikin and haptic device [64, 65]. b) Färber (2009) used stereo graphics and haptics $[66,67]$.

Färber et al. created a lumbar puncture simulator with anatomical graphics, stereographic display and a haptic device (Fig. 9b), $[65,66]$. A Sensable Technologies Phantom Premium 1.0 haptic device with six degrees of freedom is used to steer the virtual needle and allows force feedback in 3D with rotation and transversal needle movement. The needle movement is improved by restricting rotation and transversal motion of the needle once inside of the body. Haptic forces are calculated from segmented data and original CT scans, by using a haptic volume-rendering approach. The 3D virtual patient is built from data from the Visible Human data set and CT scans from real patients. The visual display consists of a 
3D virtual anatomy with moving needle, plus three 2D views showing orthogonal slices (transversal, sagittal, and frontal) of the image data. The whole scene can be rotated, panned and zoomed using the mouse. Stereo-view can be generated with shutter or anaglyph glasses to improve the impression of depth in the virtual body. A 'needle cam' can be used to show the scene from the perspective of the user-steered virtual needle. Patient variation consists of three virtual patients each with different force settings, but obesity parameter is not changeable. Body The system has been evaluated in a pilot study with a good outcome; medical students who received training on this system performed better than those who did not [65]. Due to these technologies, this virtual reality system cost considerably more to construct than other simulators.

Infant-sized manikin-based simulators have also been developed for practicing lumbar puncture (Fig. 10a-c). The Lifelform ${ }^{\circledR}$ Pediatric Lumbar Puncture Simulator (Nasco, Fort Atkinson, Wisconsin, USA) allows training for infants that require lumbar puncture [67]. The dural sac contains simulated CSF with an embedded iliac crest and removable spine, spinal canal and skin pad. The Kyoto Kagaku Co., Ltd (Japan) Paediatric Lumbar Puncture Simulator has been designed to enhance formal lumbar puncture procedural skills training and assessment [68]. Closely simulating the lumbar spinal anatomy of a 7-10 month old infant it includes palpable anatomical landmarks and aims to provide life-like sensation of both skin and tissue resistance to the spinal needle. Simulated CSF can be collected and the pressure measured. Baby Stap (Nasco, Fort Atkinson, Wisconsin, USA) is a replica of a neonatal infant of low birth weight, designed for the practice of lumbar puncture techniques [69]. Lateral decubitus position and upright are both supported. An interchangeable spine with spinal cord may be palpated for location of correct puncture site and fluid can also be infused to mimic CSF.
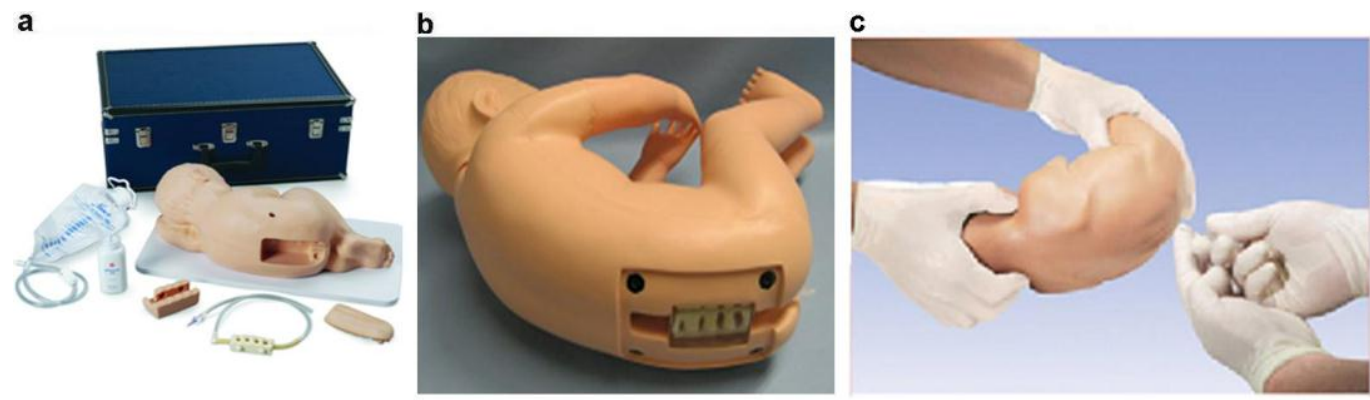

Fig. 10. Pediatric lumbar puncture simulators: a) Life/form ${ }^{\circledR}$ Pediatric Lumbar Puncture Simulator reproduced with permission from Nasco $₫ 2012$ b) Pediatric Lumbar Puncture Simulator, reproduced with permission from Kyoto Kagaku Co. Ltd. c) Baby Stap, reproduced with permission from Nasco (C) 2012 


\section{Comparative Assessment}

The general differences between the manikin simulators and the computer-based simulators are quite marked and there is a clear boundary between these two categories, with each having their own set of advantages and disadvantages as shown in Table 2 and 3. Manikin simulators have the advantage of looking similar to a patient. They are easy to set up, portable and usually cheaper. The main advantage is that manikins are physical rather than virtual, and since a clinician uses his/her hands to perform the epidural insertion a physical simulator may be a closer analogy. For example, the manikins often contain a saline-filled spinal canal which can leak if the dura is punctured and the operator can remove the skin and look inside at the physical parts, which helps to envisage the spinal anatomy. One disadvantage of manikin simulators is that the accuracy of the needle insertion forces through artificial rubber or plastic skin is hard to measure. The majority of insertion forces have been designed iteratively by opinions from clinicians trying an insertion and giving feedback, but it is not based on actual recorded data. Moreover, it is difficult for manikins to contain all of the correct anatomical layers such as skin, subcutaneous fat, various ligaments, meningeal layers and bone. Lack of ability to accommodate for patient variation such as obesity and pregnancy is another particular difficulty faced by some manikin models such as the Thoracic \& Lumbar Epidural Anaesthesia Simulator (Limb \& Things, Bristol, UK), Adult lumbar puncture simulator (Shengyuan Medical Devices Co., Ltd, Sichuan Province, China) and the Adult Lumbar Puncture Simulator (Medi-Tech, Berkshire, UK) and Life/form ${ }^{\circledR}$ Spinal Injection Simulator (Nasco, Fort Atkinson, Wisconsin, USA). As well as looking less realistic compared with other simulators, they are also limited by fewer interspinous spaces at which to practice insertion.

Limited studies following the introduction of computer-based simulators do seem to suggest a general acceptance and no evidence that they are deemed less user-friendly than the manikin models. The computer-driven simulators have two main benefits; haptic technology and real time 3D visualisations. Haptic devices have greatly improved over recent years and are verifiable to exert accurate forces within a small margin. Twelve studies [24, 33-34, 49-51, 72, 75-79] have measured needle insertion force from a variety of tissues including canine, porcine, post-mortem human and live human volunteers. This force data describes the forces encountered on the Tuohy needle as it is inserted. The aim of haptic devices is to recreate forces to simulate the ligaments, tissues and bone. The benefit of 3D virtual reality is that it allows the needle to be seen from all angles and its location in relation to internal spinal anatomy can be clearly visualised. It allows for 
limitless needle insertions without leaving marks from previous attempts and the feel is not compromised by the effect of multiple attempts to the physical manikin structure. It also allows various approaches to the epidural space including midline and paramedian approaches at multiple spinal levels. Computer models can record and playback epidural attempts and give automatic feedback on results and a scoring.

Early models, such as the Bristol Epidural Simulator, were disadvantaged by graphics made from large pixels on a $24 \mathrm{x}$ 80 pixel screen and a 2D display screen. As technology improved the images displayed dramatically improved but this highlights one of the other main pitfalls and that is the lack of an actual physical manikin. Dang et al.'s early simulator, Epidural Injection Simulator (Flinders Meditech), Mediseus Epidural Simulator (MedicVision), EpiSim (Yantric Inc) and the Spinal Anaesthesia Simulator are examples of models where the spinal anatomy is not palpable. The technology is limited to only a fixed needle insertion point, more often between lumbar vertebrae 4 and 5. In fact, the EpiSim model only has two vertebrae in the design. Being able to physically palpate the spinal landmarks, such as the iliac crests and spinous processes is the initial key step when preparing to insert an epidural and this is often challenging in the obese for example. It allows the correct spinal level to be identified in which to site the epidural catheter but it also begins to create in one's mind a 3D image of the spine as a whole, rather than just an isolated segment. This can identify potential difficulties such as spinal conditions, or slight curvatures such as kyphoscoliosis in the spine or perhaps even a slight rotation of the sitting patient. Moreover, location of the midline, especially in the obese, is one of the more challenging aspects of epidural anaesthesia but this aspect cannot be practiced if the needle position is fixed. Computer-based models also tend to cost significantly more than the manikin only models.

Table 2. A comparison of 17 manikin-based epidural simulators. 


\begin{tabular}{|c|c|c|c|c|c|c|c|c|c|c|}
\hline & 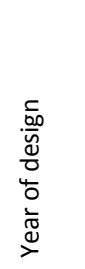 & 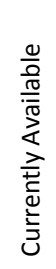 & 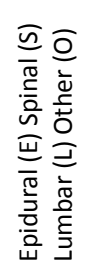 & 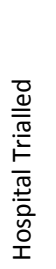 & 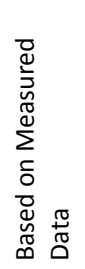 & 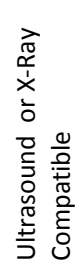 & 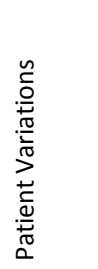 & 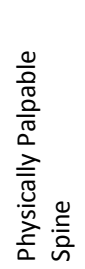 & 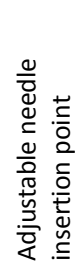 & 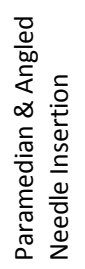 \\
\hline SimULab Lumbar Puncture / Epidural & 2008 & $\sqrt{ }$ & EL & & & $\sqrt{ }$ & 4 Skins & & & \\
\hline Blue Phantom LP and Spinal Epidural & 2011 & $v$ & ELO & & & $\sqrt{ }$ & 2 & & & \\
\hline M43B Lumbar puncture simulator-II & 2010 & 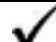 & ES & $\sqrt{ }$ & & & 3 Skins & & & \\
\hline Nasco Life/form ${ }^{\circledR}$ Spinal Injection Sim & 2008 & & ESLO & & & & 3 & & & \\
\hline Regional Anaesthesia Sim Manikin & 2007 & & ESLO & & & $\sqrt{ }$ & 1 & & & \\
\hline Pharmabotics Lumbar Epidural Inj. L.P. & 2008 & $\checkmark$ & ESL & & & & 1 & & & \\
\hline Thoracic \& Lumbar Epidural & 2007 & $\checkmark$ & $E$ & & & & 1 & & & \\
\hline Greengrocer model & 1980 & & $\mathrm{E}$ & & & & 1 & & & \\
\hline Shengyuan Lumbar Puncture Sim & 2008 & $\sqrt{ }$ & ESL & & & & 1 & & & \\
\hline Medi-Tech Lumbar Puncture Sim & 2007 & 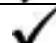 & ESL & & & & 1 & & & \\
\hline Adam, Rouilly & 2011 & $\sqrt{ }$ & ESLO & $\sqrt{ }$ & & $\sqrt{ }$ & 1 & & & \\
\hline Duggan patent & 2000 & & E & & & & 1 & & & \\
\hline Kurosaki patent & 2002 & & $\mathrm{E}$ & & & & 1 & & & \\
\hline ESTA & 2003 & & $\mathrm{E}$ & & & & 1 & & & \\
\hline Nasco Life/form ${ }^{\otimes}$ Pediatric L. P. Sim & 2008 & $\sqrt{ }$ & $\bar{L}$ & & & & 1 & $\sqrt{ }$ & & $\sqrt{ }$ \\
\hline Paediatric Lumbar Puncture Simulator & 2008 & $\checkmark$ & $\mathrm{L}$ & & & & 1 & & & \\
\hline Baby Stap & 2009 & $\sqrt{ }$ & $\mathrm{L}$ & & & & 1 & & & \\
\hline
\end{tabular}

Table 3. A comparison of 14 computer-based epidural simulators.

\begin{tabular}{|c|c|c|c|c|c|c|c|c|c|c|c|c|}
\hline & $\begin{array}{l}\frac{5}{d 0} \\
\frac{.0}{0} \\
\frac{d}{0} \\
\frac{0}{0} \\
\frac{1}{0} \\
\stackrel{0}{0}\end{array}$ & 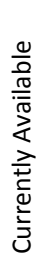 & 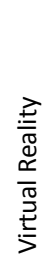 & 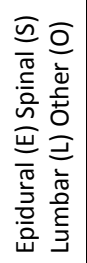 & 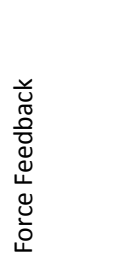 & 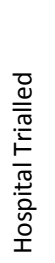 & 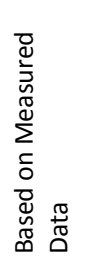 & 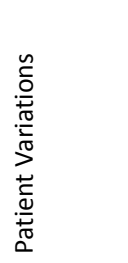 & 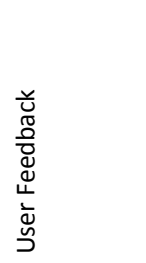 & 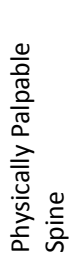 & 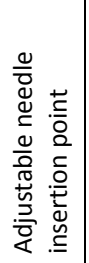 & 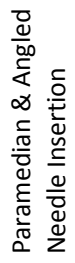 \\
\hline Spinal Anaesthesia Simulator & 2006 & & $3 D$ & ES & Phantom & & $\mathrm{CT}+$ & 1 & Knowledge & & & \\
\hline EIS (Epidural Injection Simulator) & 2003 & & & $\mathrm{E}$ & Custom & & & 6 settings & Rapid Skill & & & \\
\hline Hiemenz, Stredney, Epidural Sim & 1995 & & $3 D$ & $E$ & Yes & & Porcine & Multiple & & & & \\
\hline Mediseus epidural simulator (MES) & 2006 & & $3 D$ & $\mathrm{E}$ & Custom & & & 2 & Curriculum & & & \\
\hline Physical Sciences Inc Epidural Sim & 2010 & & $2 \mathrm{D}$ & $E$ & Actuator & & & 3 settings & & & & \\
\hline EpiSim by Yantric Inc & 2008 & & $3 D$ & $E$ & Phantom & & & Scrollbars & & & & \\
\hline Brett's Needle Insertion Simulator & 1997 & & & $E$ & Actuator & & Yes & 1 & & & & \\
\hline Dang's 2001 epidural simulator & 2001 & & & $E$ & Phantom & & & Scrollbars & & & & \\
\hline Bostrum's Lumbar puncture Sim & 1993 & & $3 D$ & $\mathrm{~L}$ & Actuator & & & 1 & & & & \\
\hline Färber Lumbar puncture Simulator & 2009 & & $3 D$ & $\mathrm{~L}$ & Phantom & & CTScan & 3 & Recordings & & & \\
\hline Gorman Lumbar Puncture Simulator & 2000 & & $3 D$ & $\mathrm{~L}$ & Phantom & & & 1 & Recordings & & & \\
\hline Bristol Epidural Simulator & 1998 & & $2 \mathrm{D}$ & $E$ & Custom & & & 1 & Results & & & \\
\hline Bacon epidural injection simulator & 1990 & & & $E$ & Spring & & & 1 & & & & \\
\hline Gillespie Virtual Epidural Simulator & 1995 & & & $E$ & Custom & & CTScan & 1 & & & & \\
\hline
\end{tabular}




\section{Technology and Implementation}

\subsection{Commercial haptic devices}

Many of the reviewed haptic epidural simulators use commercially available haptic devices. SensAble Technologies created the Phantom Desktop, Phantom Premium 1.0 - 1.5 and Phantom Omni. Some simulators have also used Novint Falcon. The devices can be modified with needle-shaped stylus attachments (Fig. 11). Coles et al. described electrical modification of a phantom omni device to attach a needle stylus which involved removing panels and replacing some of the potentiometers and circuitry [70,71]. These off-the-shelf haptic devices are usually shipped with an Application Programming Interface (API) which offers support for software development in various programming languages. The Spinal Anaesthesia Simulator contains software which uses the H3D API2 and the volume haptics toolkit VHTK, which provide support for controlling phantom desktop. The API for Novint Falcon is similar. Software can specify a 3D vector $(\mathrm{x}, \mathrm{y}, \mathrm{z})$ representing the force in each dimension on a scale of 0 to 1 . These forces can be applied selectively dependent upon the 3D location of the stylus, which enables different forces to be generated for each layer of tissue.
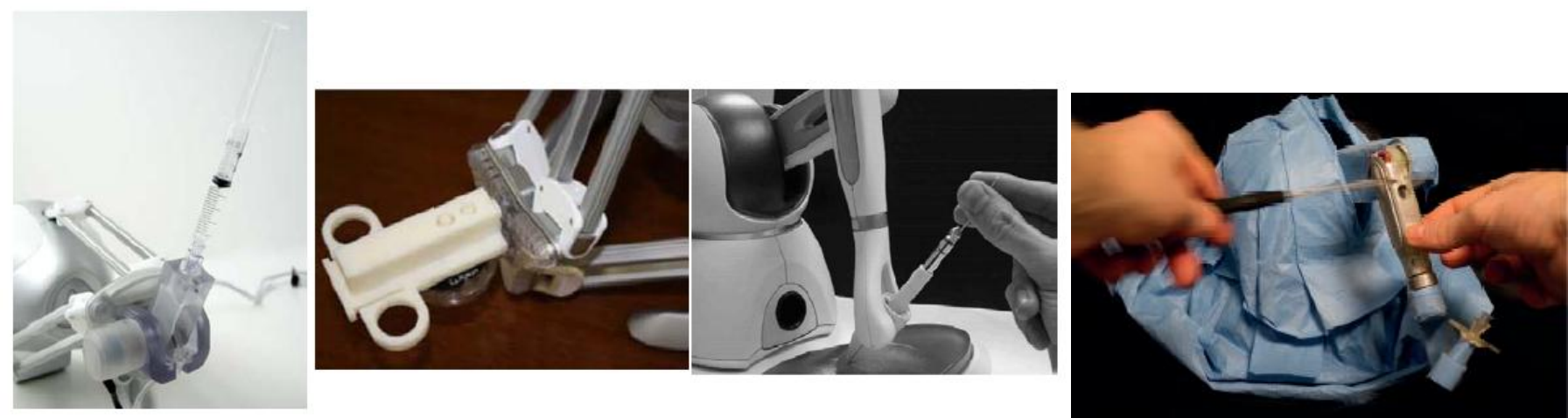

Fig. 11. Modified haptic attachments designed for use in needle insertion and modification of a Phantom Omni (Coles 2011) [70, 71].

\subsection{Custom-made haptic devices}

Some epidural simulators have custom built haptic devices incorporated. One such example from Physical Sciences Inc. (PSI), (Massachusetts, USA) uses a novel actuator proposed by Magill et al. [72-74]. The actuator uses a set of cables to suspend a needle holder. The cables are wound onto spools controlled by pulley motors. An electromagnetic tracker is used to monitor the position of the needle tip (Fig. 12a). The simulator reproduces properties of multilayer tissue structures. Needle insertion force peaks at about $6 \mathrm{~N}$, which is a similar force to that of a real patient [73]. Anaesthetists 
have tested the system and found that the tactile feedback is good but mechanical vibrations need further refinement. Median and paramedian insertions are supported. Patient variations include age, sex and obesity. Electronic machinery is required to drive the control motors and a transmitter. Many cables connecting various components make the device less portable.

a

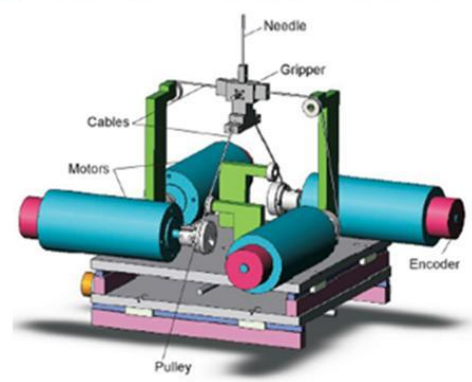

b

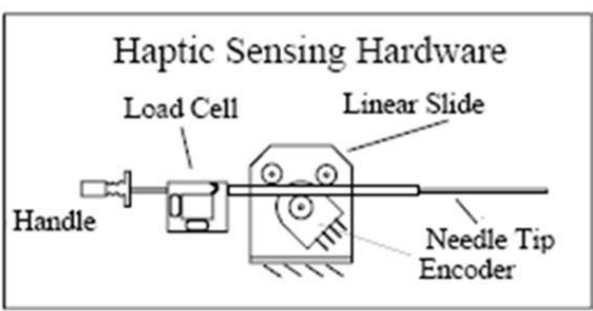

b

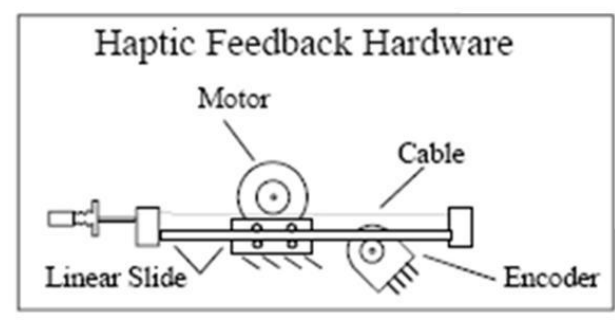

Fig. 12. a) Custom designed haptic actuator by Magill (2010) [72-74] b) Two haptic devices by Gillespie (1995) [27].

Gillespie et al. created novel custom built haptic sensing and haptic feedback devices (Fig. 12b) [27]. The haptic sensing device was used to obtain data from tissue samples to generate force models. The haptic feedback device alone makes up the simulator which recreates the force models. The haptic sensing device was made from an epidural needle fitted with a custom built load cell. The needle is constrained to linear motion by bearings. An amplifier PCB, A/D converter and I/O card allows connection to a computer for data collection. The haptic feedback device is made up of a linear slide motorized through a capstan drive by a low-inertia precision motor. An encoder is used to track position of the slide. To minimize inertia, the rod itself moves and the platform is fixed. The author reported the device to be inaccurate because the mass of the sensing device on the needle masks some of the feeling of needle insertion when testing on a pear and tomato. They stopped using the physical device and reverted to using a perceptual model for training.

\subsection{Needle force data}

Haptic devices work by generating force based on numerical data. The accuracy of the device depends on the accuracy of this data, so it is vital that the data is collected from actual in vivo measurements. Previous simulators have adjusted forces by opinion of anaesthetists, but this qualitative method is prone to error, individual bias and is less representative of the in vivo situation. An alternative by Gillespie et al. was to measure forces using a load cell on the needle [27]. Tran et al. collected force data using a force sensor on the plunger of the syringe, under the operator's glove [24]. Hiemenz et al. collected insertion force data from porcine, canine and human tissue models [75]. Twelve studies [24, 33-34, 49-51, 72, 
75-79] have measured the forces during Tuohy needle insertions. It is important that measured force data is incorporated into the haptic device. Ideally, a range of patients should be modelled to give variation, so this will require future studies to obtain measurements from patients to obtain the necessary data.

\subsection{Liquid flow}

In robotic simulators, such as Bristol, Mediseus and PSI there is an internal reservoir which collects the saline at loss of resistance (LOR). Often this is implemented with an electronic solenoid valve which is controlled electronically by the software and opened at the correct depth to signify LOR (Fig. 13). In manikin simulators such as Nasco's Life/form ${ }^{\circledR}$ Spinal Injection Simulator, Limb \& Things’ M43B Lumbar Puncture Simulator-II, and Pharmabotics' Lumbar Epidural Injection and Lumbar Puncture Trainer liquid flow from the syringe is prevented by the rubber skin material, but as the needle pierces through, the liquid can escape as occurs in vivo. Manikin simulators also provide CSF liquid flow from an accidental dural puncture. CSF leak is simulated by a saline bag which fills a spinal canal with liquid. The spinal canal is made from a synthetic rubber material which when punctured causes liquid to siphon out and as the needle is withdrawn the rubber material closes the puncture and tends to mostly stop the flow. This can then be re-used many times before a replacement spinal canal is installed and re-filled with saline.

\subsection{Stereoscopic $3 D$}

Several options for stereo 3D have been explored by the above simulators. Polarised 3D glasses, used by Hiemenz et al. create 3D illusion by restricting the light to each eye and can be full colour [75]. Immersive haptic workbenches, used by Färber et al. and 3D monitors work by having two separate screens at 90 degree angles and a 45 degree glass plate which reflects half of the light, allowing the two images to combine $[65,66]$. Another option is Anaglyph images, built from red and cyan filters on left and right eye which enable 3D imaging but full colour cannot always be recreated. 3D video software such as Unity or Quad Buffer OpenGL could be used in future to interface with 3D displays. Stereoscopic 3D imagines can create or enhance the illusion of depth by displaying two separate images from offset angles. This may give

a more intuitive visualisation of depth when inserting the needle. Conventional 3D allows the needle to be seen from various angles, however there is no way to know the actual distance between objects other than by estimating their size. Stereo uniquely enables the viewer to perceive depth, which could be a more intuitive way to visualise distance of insertion in relation to internal anatomy during insertion. Depth judgment is crucial to the epidural procedure and since 
stereo graphics allows the perception of depth in 3D graphics against a benchmark, epidural simulators can benefit greatly from stereo technology.

\subsection{Artificial skin}

Some of the reviewed simulators recreate the feel of skin with a skin plate made from synthetic material. The PSI epidural simulator uses a block of artificial skin made from two layered rubber (Fig. 14). A similar synthetic material is used to represent skin in most of the manikin simulators and can be purchased separately as replacements or to modify the tissue density giving patient variation. The ESTA simulator used layers of carbon-fibre electrically-conductive fabric in between artificial tissue layers, which allows electrical detection of needle location without a wire being attached to the Tuohy needle [80]. The conductive fabric used was Gorix although the production has now ceased.

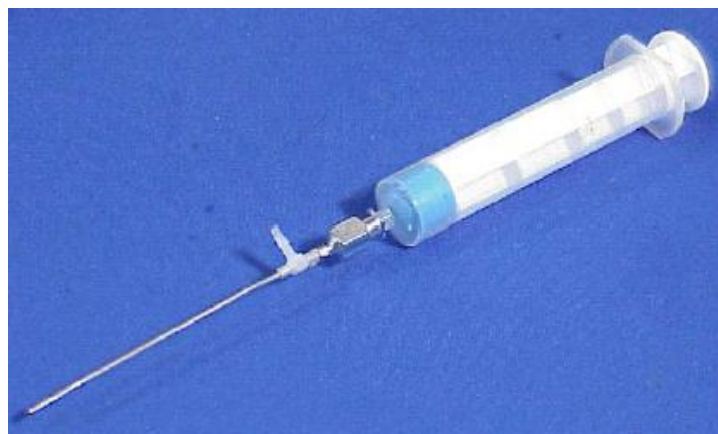

Fig. 13. Solenoid valve allows electrical control of liquid flow (Magill

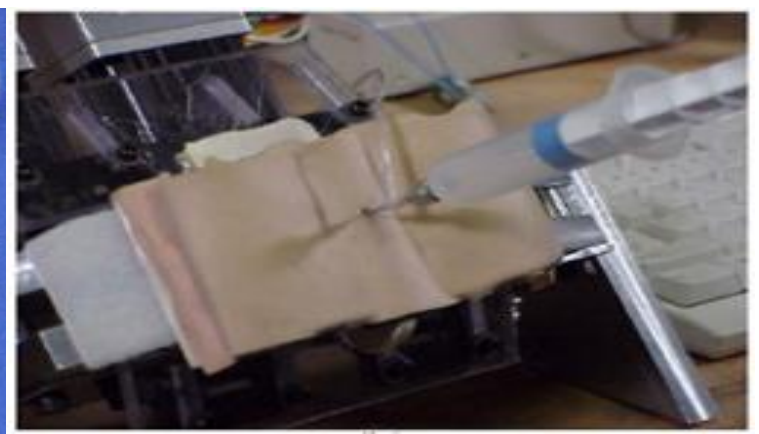

Fig. 14. Artificial Skin from the PSI epidural simulator [73-74] 2004) [73-74]

\section{Proposal for an Ideal Simulator}

The ideal epidural simulator should combine the successful features of previous simulators and aim to recreate as far as possible the in vivo procedure. The reason why manikin and computer-based simulators are divided as such is because there is difficulty combining the two. For example, if real materials were used simultaneously with a haptic feedback device, the materials would affect the generated feedback forces. In industry, an example of a gold standard simulator is the flight simulator cockpit used for simulation, training and testing of pilots where the flying experience mimics actual flying to a high degree [81]. Advanced flight simulation environments such as the semi-virtual cockpit have led to flight training simulations being adopted by major airlines universally, and their effectiveness is well recognized. For epidural simulators, this would be a combination of technology from computer-based simulators and manikin-based models. It 
would have a human-shaped physical form containing vertebrae with spinous processes that are palpable. Ultrasound compatibility would be supported for visualisation of the inter-vertebral gap between spinous processes, the lamina, ligamentum flavum and epidural space. Ultrasound scanning would be useful for localising the epidural space in obese patients where the bony landmarks are less easily palpable. A real Tuohy needle could be inserted at any intervertebral space in the lumbar or thoracic region using the midline or paramedian approach. It would contain a force feedback haptic device, with force data originating from measured Tuohy needle insertions from patients. The forces would automatically adjust to give patient variation on weight, height- and sex. This could simulate random patients or match measurements from a specific patient. The 3D virtual patient and virtual vertebrae will also need to adjust in size and shape to match measurements from actual patients. As the needle advances, forces would represent each tissue layer and a LOR on reaching the epidural space. Once the epidural space is reached, saline would be released by a solenoid into an internal reservoir. Existing simulators have model LOR as $100 \%$ loss of resistance. In vivo it may be the case that resistance during insertion is not always either $100 \%$ or $0 \%$ but somewhere in between. If this could be measured in a clinical trial, partial resistance could be incorporated into future epidural simulators, to further convey the full information of a real epidural. During the entire insertion, a 3D virtual spine would be displayed on the monitor showing the moving needle's position in real time. The manikin could bend forwards to mimic spinal flexion to increase spacing between the vertebrae.

Variation in patient size, height, weight-and other characteristics should be possible based upon actual patient measurements. Currently, most simulators have only two or three options such as obese, elderly and normal which is perhaps not enough to encapsulate reality and could therefore be improved. Simulators could have unlimited patient variation by including parameters such as height, weight, body shape-and obesity which could be adjustable. Ideally, the settings should match to measurements from real patient data. The adjustments could happen automatically based upon basic patient data, so that the user does not have to manually configure all the settings. The simulator could then re-create a virtual model of a particular patient. Clinicians planning on performing the epidural can practice beforehand on a virtual model of the patient thereby reducing the learning curve during the procedure on the patient. The four common patient positions adopted during epidural insertion are sitting, sitting with lumbar flexion, lateral decubitus and lateral decubitus with lumbar flexion. These four common positions at least should be modelled in an epidural simulator to give a greater level of realism than static epidural simulators. Ideally, variable spine flexibility could be achieved by modelling 3D 
flexible spine vertebrae. This may allow simulation of spinal conditions such as curvatures caused by scoliosis. These conditions cause difficulties in placing the needle due to unusually positioned landmarks.

Assessment of trainees' skill level and performance feedback would be a real advantage in the ideal simulator. When epidural simulators are used as part of an assessment, the application of structured assessment criteria such as Knowledge Space Theory (KST), or a curriculum-based assessment can provide a framework for objective feedback. If assessment criteria are applied, simulators could be used as part of training as a Simulated Interactive Learning System (SILS). The user should be able to choose the needle insertion point themselves because that is a key feature when performing a real insertion. Some current computer simulators do not allow this because of limitation on the haptic devices. Also the needle angle and approach (midline or paramedian) should be chosen by the user.

Palpability of the spine should be possible and this is why having a manikin is essential. However, the manikin can also be designed to have 'additional layers' to simulate obesity or a retractable spine from soft tissues where the spinous processes are not easily palpable. Simulators which allow the use of ultrasound to determine the position of the spinal landmarks would be advantageous in these cases. Anatomical landmarks guide anaesthetists to suitable intervertebral levels in which to place the epidural or spinal, either to minimise possible neurological damage to the patient or to achieve satisfactory analgesic block at the necessary dermatomal levels. Haptic guidance allows a user to replay an insertion as performed by an expert. This would benefit the learner by demonstrating an acceptable insertion speed and the resistive forces to expect. Voice recognition has been incorporated into some simulators and avoids having any keyboard or mouse manual configuration - this approach is equally welcome.

Finally, none of the simulators to date take into account the environmental challenges of inserting an epidural and this is especially true for obstetric anaesthesia. In this scenario, the woman is often a 'moving target' especially during labour contractions and there is the sound of the cardiotocograph (fetal heartbeat and the uterine contractions during pregnancy) recordings and other people to contend with. In an ideal simulator, these additional environmental or situational matters could be incorporated by use of a moving base for the simulator and earphones of actual recordings of the noise in a birthing room with the woman giving feedback to the anaesthetist when she has a contraction or is in pain. The above will 
go some way in imitating the training simulators employed by the aviation industry, whereby the simulated flight cockpit gives a real sense of flying an actual plane in differing weather conditions.

From this review, these are the proposed characteristics of an ideal epidural simulator: (1) integrated physically palpable parts as well as computer based visualisation (2) simulator configurable on measured patient data (3) model updatable based on basic patient data (4) incorporation of body mass index (BMI) and body shape into the model, although BMI is not necessarily related to increased needle depth due to fat above the spine. (5) possible to interpret CT and MRI data readings and update into simulator (6) flexible spine allowing for lumber flexion and lateral decubitus or sitting position (7) parametric modelling of graphics (8) palpation through the physical manikin and haptic feedback (9) recording of ideal insertion and playback (10)voice input/output for interaction and control (11) performance evaluation and feedback to user and (12) immersive simulation through use of 3D visualisation and artificial sound.

\section{Conclusions}

There are difficulties measuring data for all forces and torques on the needle and plunger of an epidural simulator preventing the use of actual measurement within the apparatus. These difficulties cause problems for both computer-based and manikin-based simulators. Additionally there are limitations to haptic devices including their limited resolution, dynamic movement range, force range and response rate. Further studies may be required to prove whether haptic devices are capable of more accurate force simulation than layers of artificial tissue. Haptic devices do possess features which cannot be achieved by synthetic tissue layers that suggest they may be more suitable, including the generation of torque, the ability to finely-tune force feedback and ability to blend forces between layers.

Manikin models are easier to set up, more portable, easier to clean and are often less expensive. Manikins are physical rather than virtual, and since a clinician uses his/her hands to perform the epidural insertion, a physical simulator may be a closer analogy to a patient from the tactile point of view. The user can see the physical body and choose where to insert the needle. However, the manikin simulators are limited by their composition. Users cannot view the needle location inside the body without disassembling the model, whereas needle position can be viewed in real time on computer simulators. It is not known how accurately the rubber plates mimic the densities of skin, tissues, ligaments and bone. The rubber plates and parts are generally self-healing but they do degrade over time due to holes from previous insertions and 
so require costly replacement parts. Conversely, haptic devices have an indefinite lifespan. Patient variation such as obesity and body shape can only be supported through interchangeable skin plates which often cost extra and this limits the models to a finite number of variations. It is occasionally unclear as to whether the procedure has been successful or not, which becomes important when these are used as examination tools for students. Success or failure is less distinct and subjective to the examiner's opinion, whereas electronic or computer-driven devices can confirm accurate placement. Computer driven simulators can give a summary of the trainees performance can be summarized at the end of the training session and scored and this can be compared with their peers to assess level of competency. Therefore incorporation of both features may be beneficial which may be used separately or in combination.

Haptic devices have become precision scientific instruments which can exert forces and torques in three dimensions. Twelve studies [24, 33-34, 49-51, 72, 75-79] have measured needle insertion force from a variety of tissues including canine, porcine, post mortem human and live patients. The aim of using haptic devices was to attempt to recreate measured forces to represent the ligaments, tissues and bone; however there are many difficulties with such measurement of data. It is not possible to measure all forces and torques on both needle and plunger in vivo, it is hard to replicate in vivo measurements on cadaveric tissue samples because the presence of blood significantly affects needle friction, and porcine or canine models vary from human tissue. There are challenges understanding the differences between static and dynamic forces and torques, friction and static friction. Haptic devices also have limitations with the resolution, dynamic range and speed which have adverse affect when attempting to mimic needle insertions. Computer models can record and playback an expert's insertion, or give automatic feedback guidance on progress, which manikins cannot. Computer models are easier to adjust than manikin models, allowing patient variations such as obesity and tissue changes in the elderly, with no extra parts or cost, by using scrollbars to adjust settings. 3D computer visualisations allow the user to monitor during insertion the location of the needle amongst the tissues and vertebrae. Computer insertions can be made many times without degrading or leaving marks from previous attempts. Voice recognition and voice feedback are another advantage to computer models. However, with computer-based simulators, the needle is often in a fixed position or orientation, which does not allow practice of palpation or needle positioning, although several manikin simulators also have a fixed needle position. Some simulators are a combination of the two - a computer-driven simulator but also with a manikin and these in theory should be superior because they possess the benefits of both types. 
Comparisons and reviews of the existing epidural and spinal simulators have been made, outlining the positive and negative aspects of each. Existing simulators have shown impressive features but there is still the potential for further improvement as illustrated when considering the ideal characteristics of epidural and spinal simulators. If the positive aspects could be drawn together to create such a simulator, this would undoubtedly be of great benefit in the clinical setting in making the epidural procedure safer and reducing patient morbidity. The case for developing a high fidelity epidural simulator is overwhelming.

\section{Declarations}

Competing interests: None declared

Funding: None

Ethical approval: Not required

\section{References}

[1] Catchpole K, Bell D, Johnson S, Boult M. Reviewing the evidence of patient safety incidents in anaesthetics. Internal Report. The National Patient Safety Agency 2006.

[2] Cook TM, Counsell D, Wildsmith JAW. Major complications of central neuraxial block: report on the Third National Audit of The Royal College of Anaesthetists. Br J Anaesth 2009;102:179-90.

[3] Paech MJ, Godkin R, Webster S. Complications of obstetric analgesia and anaesthesia: a prospective analysis of 10,995 cases. Int J Obstet Anaesth 1998;7:5-11.

[4] Jenkins JG. Some immediate serious complications of obstetric analgesia and anaesthesia: a prospective study of 145,550 epidurals. Int J Obstet Anaesth 2005;14:37-42.

[5] Gleeson CM, Reynolds F. Accidental dural puncture rates in the UK. Int J Obstet Anaesth 1998;7:242-6.

[6] Gupta S, Collis R, Harries S. Increasing dural tap rate: is this a national trend? Int J Obstet Anaesth 2007;16:S17.

[7] Naik VN, Devito I, Halpern SH. Cusum analysis is a useful tool to assess resident proficiency at insertion of labour epidurals. Can J Anesth 2003;50:694-8.

[8] Konrad C, Schupfer G, Wietlisbach M, Gerber H. Learning manual skills in anesthesiology: is there a recommended number of cases for anesthetic procedures? Anesth Analg 1998;86:635-9. 
[9] Watterson LM, Hyde S, Bajenov S, Kennedy SE. The training environment of junior anaesthetic registrars learning epidural labour analgesia in Australian teaching hospitals. Anaesth Intensive Care 2007;35:38-46.

[10] Grantcharov TP, Reznick RK. Teaching procedural skills. Br Med J 2008;336:1129-31.

[11] Smith TS, Johannsson HE, Sadler C. Trials of labour: can simulation make a difference to obstetric anaesthetic training? Curr Anaes Crit Care 2005;16:289-96.

[12] Aggarwal R, Darzi A. Technical skills training in the $21^{\text {st }}$ century. N Engl J Med 2006;355:2695-6.

[13] Bould MD, Crabtree NA, Naik VN. Assessment of procedural skills in anaesthesia. Br J Anaesth 2009;103:47283.

[14] Szypula K, Ashpole KJ, Bogod D et al. Litigation related to regional anaesthesia: an analysis of claims against the NHS in England 1995-2007. Anaesthesia 2010;65:443-52.

[15] Dogliotti AM. Research and Clinical Observations on Spinal Anesthesia: With Special Reference to the Peridural Technique. Anesthesia and Analgesia 1933;12:59-65.

[16] Wong DT, Prabhu AJ, Coloma M, Imasogie N, Chung FF. What is the minimum training required for successful cricothyroidotomy? Anesthesiology 2003;98:349-53.

[17] Working Party of the Association of Anaesthetists. Consent for anaesthesia, revised edition. London, AAGBI 2006.

[18] Royal College of Anaesthetists. Curriculum for a CCT in Anaesthetics - version 2. London, Aug 2010.

[19] Bruce RCH, McLeod ADM, Smith GB. A survey of UK anaesthetic trainee attitudes towards simulator based training experience. Bulletin of the RCOA 2005;34:1722-3.

[20] Gaba DM. Improving anesthesiologists' performance by simulating reality. Anesthesiology 1992;76:491-4.

[21] Zausig YA, Bayer Y, Hacke N et al. Simulation as an additional tool for investigating the performance of standard operating procedures in anaesthesia. Br J Anaesth 2007;99:673-8.

[22] Kneebone RL, Nestel D, Vincent C, Darzi A. Complexity, risk and simulation in learning procedural skills. Med Educ 2007;41:808-14.

[23] Riley R, Wilks D, Freeman J. Anaesthetists' attitudes towards an anaesthesia simulator. A comparative survey: USA and Australia. Anaesth Intensive Care 1997;25(5):514-9.

[24] Tran D, Hor K, Kamani A et al. Instrumentation of the loss-of-resistance technique for epidural needle insertion. IEEE Trans BioMed Eng 2009;56:820-7. 
[25] Vidal FP, John NW, Healey AE, Gould DA Simulation of ultrasound guided needle puncture using patient specific data with 3D texture and volume haptics. Comp Anim Virtual Worlds 2008;19:111-127.

[26] Abolhassani, N. Patel, R. Moallem, M. Needle insertion into soft tissue: A survey. Medical Engineering \& Physics 2007;29(4):413-431.

[27] Gillespie B, Rosenberg LB. Design of high-fidelity haptic display for one-dimensional force reflection applications. Proc. SPIE 1995;2351:44-54.

[28] Friedman Z, Siddiqui N, Katznelson R, Devito I, Bould MD, Naik V. Clinical impact of epidural anesthesia simulation on short and long-term learning curves: high versus low-fidelity model training. Reg Anesth Pain Med 2009;34(3):229-32.

[29] Sakamaki T, Sairyo K, Sakai T, Tamura T, Okada Y, Mikami H, Measurements of Ligamentum Flavum Thickening at Lumbar Spine Using MRI, Arch Orthop Trauma Surg., 2009;129(10):1415-9.

[30] Nickalls RWD, Kokri MS, The width of the posterior epidural space in obstetric patients, Anaesthesia, 1986;41:432-433.

[31] Putz R, The detailed functional anatomy of the ligaments of the vertebral column, Annals of anatomy, 1992;174:40-47.

[32] Prestar FJ, Putz R, Das Ligamentum longitudinale posterius - Morphologie und Funktion, Morphol Med, 1982;2:181 - 189.

[33] Hiemenz L, Force models for needle insertion created from measured needle puncture data, Medicine meets virtual reality, 2001:180-6.

[34] Vaughan N, Dubey VN, Wee MYK, Isaacs R, Haptic Interface on Measured Data for Epidural Simulation, Proceedings of the ASME 2012 International Design Engineering Technical Conference and Computers and Information in Engineering Conference, DETC2012-70891, August 12-15, Chicago, IL, USA.

[35] Leighton BL. A greengrocer's model of the epidural space. Anesthesiology 1980;70:368-9.

[36] Limbs \& Things, Bristol, UK, 2011. http://limbsandthings.com/uk/products/thoracic-lumbar-epidural-anesthesiasimulator/ (accessed 12/04/2012)

[37] Pharmabotics Ltd, Lumbar Epidural Injection and Lumbar Puncture Trainer http://www.pharmabotics.com/lumbar-epidural-injection-and-lumbar-puncture-trainer/ (accessed 12/04/2011).

[38] Nasco, Life/form ${ }^{\circledR}$ Spinal Injection Simulator, http://www.enasco.com/product/LF01036U (accessed 12/04/2012) 
[39] Kyoto Kagaku, Japan. Simulators for medical education, 2011.

http://www.kyotokagaku.com/products/detail01/m43b.html (accessed 12/04/2012)

[40] Uppal V, Kearns R, McGrady E. Evaluation of M43B Lumbar puncture simulator-II as a training tool for identification of the epidural space and lumbar puncture. Anaesthesia 2011;66:493-6.

[41] National Institute for Health and Clinical Excellence. Ultrasound-guided catheterization of the epidural space. [IPG249]. London, National Institute for Health and Clinical Excellence, 2008.

[42] Simulab Corporation, Washington, USA. Medical training models, 2011. http://www.simulab.com/product/critical-care/lumbar-puncture-epidural-trainer (accessed 12/04/2012).

[43] Blue Phantom, Redmond USA, Ultrasound Training Models, 2011. http://www.bluephantom.com/product/Lumbar-Puncture-and-Spinal-Epidural.aspx (accessed 12/04/2012).

[44] Adam, Rouilly, Kent, UK. http://www.adam-rouilly.co.uk/products.aspx?cid=381 (accessed 12/04/2012).

[45] Duggan JE. An epidural simulator device for training practitioners in the anaesthetic technique of epidural injection. GB Patent GB2369714; 2000.

[46] Kurosaki Y. Epidural Puncture Simulator. JP Patent 2002132138; 2002.

[47] Zhangji L, Baoquan Z. Spinal puncture training simulation standard patient. Patent CN101877192; 2010.

[48] Daykin A, Bacon R. An epidural injection simulator. Anaesthesia 1990;45:235-6.

[49] Langton JA, Meiklejohn BH. Pressure generated during insertion of lumbar epidurals: a comparison with the Portex epidural injection simulator. Anaesthesia 1990;45:1055-6.

[50] Stredney D, Sessanna D, McDonald JS, Hiemenz L, Rosenberg LB. A virtual simulation environment for learning epidural anesthesia. In: Weghorst SJ, Sieburg HB, Morgan KS (eds.) Medicine meets Virtual Reality: Healthcare in the Information Age. Amsterdam: IOS Press, 1996:164-175.

[51] Brett PN, Parker TJ, Harrison AJ, Thomas TA, Carr A. Simulation of resistance forces acting on surgical needles. Proc. Inst Mech Engrs 1997;211:335-47.

[52] Dang T, Annaswamy T, Srinivasan M. Development and evaluation of an epidural injection simulator with force feedback for medical training. Stud Health Technol Inform 2001;81:97-102.

[53] Wilson JG, Pallotta OJ, Reynolds KJ, Owen H. An epidural injection simulator. World Congress on Medical Physics and Biomedical Engineering, Sydney. 2003. 
[54] CPR Savers LLC, Arizona, USA 2011. http://www.cpr-savers.com/medical-simulators/epidural-injection.html (accessed 12/04/2012).

[55] Mayooran Z, Watterson L, Withers P, Line J, Arnett W, Horley R. Mediseus epidural: full-procedure training simulator for epidural analgesia in labour. Proc. SimTecT Healthcare Simulation Conference 2006.

[56] Brien G, Horley R. Simulator and Method. Patent WO2007068050; 2007.

[57] Elks KN, Riley RH. The Mediseus epidural simulator - does it replicate real patients? Anaesth Intensive Care 2007;35:818.

[58] Dreifaldt U, Kulcsar Z, Gallagher P. Exploring haptics as a tool to improve training of medical doctors in the procedure of spinal anaesthetics. Eurohaptics conference; Paris 2006.

[59] Albert D, Hockemeyer C, Kulcsar Z, Shorten G. Competence assessment for spinal anaesthesia. HCI and Usability for Medicine and Health Care. Proceedings of the Third Symposium of the Workgroup HumanComputer Interaction and Usability Engineering of the Austrian Computer Society, USAB 2007 Graz, Austria In: Lecture Notes in Computer Science. Berlin: Springer, 2007:165-70.

[60] Yantric Inc., EpiSim, 2011 http://www.yantric.com/products.html\#episim (accessed 12/04/2012).

[61] Sensable, Simulation and Visualisation, 2011 http://www.sensable.com/industries-simulation-visualization.htm (accessed 12/04/2012).

[62] Bostrom M, Singh SK, Wiley CW. Design of an interactive lumbar puncture simulator with tactile feedback. Virtual Reality Annual International Symposium, Proceedings of the IEEE 1993:280-6.

[63] Gorman P, Krummel T, Webster R et al. A prototype haptic lumbar puncture simulator. In: Westwood JD et al. (eds.) Medicine meets Virtual Reality. Amsterdam: IOS Press, 2000.

[64] Webster R, Mohler B, Melkonian M, A haptic lumbar puncture simulator, Millersville University, http://cs.millersville.edu/ webster/haptics/lumbar/index.html (accessed 12/04/2012).

[65] Färber M, Hummel F, Gerloff C, Handels H. Virtual reality simulator for the training of lumbar punctures, Methods Inform Med 2009;48:493-501.

[66] Färber M, Hoeborn E, Dalek D et al. Training and evaluation of lumbar punctures in a VR-environment using a 6DOF haptic device. Stud Health Technol Inform 2008;132:112-4.

[67] Nasco, Life/form ${ }^{\circledR}$ Pediatric Lumbar Puncture Simulator, http://www.enasco.com/product/LF01000U (accessed $12 / 04 / 2012)$ 
[68] Kyoto Kagaku Co. Ltd, Pediatric Lumbar Puncture Simulator, http://www.kyotokagaku.com/products/detail01/pdf/m43c_catalog.pdf (accessed 12/04/2012).

[69] Nasco, Baby Stap, Simulators and Trainers, http://www.enasco.com/product/LG02094U (accessed 12/04/2012).

[70] Coles TR, John NW, Sofia G et al. Modification of commercial force feedback hardware for needle insertion simulation. Stud Health Technol Inform 2011;163:135-7.

[71] Coles TR, John NW. The effectiveness of commercial haptic devices for use in virtual needle insertion training simulations. In Advances in Computer-Human Interaction, Third International Conference 2010;148-153.

[72] Magill JC, Byl MF, Hinds MF, Agassounon W, Pratt SD, Hess PE. A novel actuator for simulation of epidural anesthesia and other needle insertion procedures. Simul Healthc 2010;5(3):179-84.

[73] Magill JC, Anderson BD, Rosen DI et al. A multi-axis mechanical simulator for epidural needle insertion. International Conference on Medical Simulation. Massachusetts; 2004.

[74] Physical Sciences Inc., 2004. http://www.psicorp.com/pdf/library/VG04-160.pdf (accessed 12/04/11).

[75] Hiemenz L, Stredney D, Schmalbrock P. Development of the force-feedback model for an epidural needle insertion simulator. Stud Health Technol Inform 1998;50:272-7.

[76] Naemura K, Saito H. Needle insertion test by porcine ligamentum flavum. World Congress on Medical Physics and Biomedical Engineering 2009, 25(VI):28-31.

[77] McKay WP, Rosser T, Kriegler S, Mohamed A. Epidural loss-of-resistance biomechanics: an open pilot cadaver study. Dove Press 2010, (3):101-7.

[78] Meiklejohn B, The effect of rotation of an epidural needle. An in vitro study, Anaesthesia 1987, 42(11):11801182.

[79] Westbrook J, Uncles D, Sitzman B, Carrie L. Comparison of the force required for dural puncture with different spinal needles and subsequent leakage of cerebrospinal fluid. Anesth Analg 1994, 79:769-72.

[80] Jones R, Pinnock C. The development of the Epidural Simulator Training Apparatus (ESTA), International Meeting for Medical Simulation, San Diego, California 2003.

[81] Gu HB, Wu DS, Liu H. Development of a novel low-cost flight simulator for pilot training. World Acad Sci Eng Technol 2009, 60:685-9. 